\title{
Epidemiología de las consultas pediátricas respiratorias en Santiago de Chile desde 1993 a 2009
}

\author{
Pedro Astudillo, ${ }^{1}$ Pedro Mancilla, ${ }^{1}$ Claudio Olmos ${ }^{1}$ y Álvaro Reyes ${ }^{2}$
}

Forma de citar

Astudillo P, Mancilla P, Olmos C, Reyes A. Epidemiología de las consultas pediátricas respiratorias en Santiago de Chile desde 1993 a 2009. Rev Panam Salud Publica. 2012;32(1):56-61.

RESUMEN Objetivo. El presente trabajo se propone describir la metodología de análisis y la distribución global de las consultas de morbilidad — particularmente las de tipo respiratorio- en menores de 15 años de edad de la ciudad Santiago de Chile, Chile, con el objetivo de conocer sus diferencias según grupos etarios, su comportamiento estacional y su evolución a lo largo de los años.

Métodos. Se investigó la distribución de las consultas de morbilidad pediátrica $(C P)$ —n especial las respiratorias-y su evolución en un período de 17 años. En siete centros centinela de Santiago de Chile se recolectó prospectivamente información diaria de todas las CP, agrupadas en infecciones no respiratorias e infecciones respiratorias agudas (IRA).

Resultados. Entre enero de 1993 y diciembre de 2009 se registraron 1947477 CP, de las cuales 1188029 (61,0\%) fueron por causa respiratoria: 656567 (33,7\%) por enfermedad respiratoria aguda de vía aérea baja (IRAb), 418932 (21,5\%) por síndrome bronquial obstructivo $(S B O)$ y 48669 (2,5\%) por neumonía. Neumonía y SBO fueron más frecuentes en menores de 5 años. Las IRAb, SBO y neumonía presentaron una significativa tendencia a la disminución durante el período observado. Las IRA constituyen la primera causa de CP en atención primaria de salud y el SBO es la primera causa específica de consulta pediátrica.

Conclusiones. Estos resultados enfatizan la necesidad de asignar o redestinar recursos en programas de promoción, educación, prevención y tratamiento de estas enfermedades, con la debida focalización que determina su variación estacional.

Palabras clave Infecciones del sistema respiratorio; vigilancia epidemiológica; pediatría; salud del niño, Chile.

La experiencia clínica indica que en Chile las enfermedades respiratorias constituyen la principal causa de consulta de morbilidad pediátrica $(\mathrm{CP})$ en atención primaria de salud (APS). No obstante hay poca literatura que avale esta impresión pues, salvo excepciones, los estudios se limitan a un solo consultorio o centro de salud o no son actuales

\footnotetext{
1 RespIRA-Chile, Santiago, Chile. La correspondencia se debe dirigir a Pedro Astudillo. Correo electrónico: pedroastudillo@vtr.net

2 Universidad Católica de Valparaíso, Santiago, Chile.
}

(1-7). Por otro lado, no hay información precisa sobre el comportamiento estacional de estas consultas. En todo caso, y con tales limitaciones, para las consultas pediátricas por causa respiratoria se han descrito cifras de promedio anual que oscilan entre $39,1 \%$ y $62,2 \%$ del total de visitas pediátricas (7).

Es razonable pensar que la distribución de las consultas no debe ser la misma en las distintas franjas etarias de la niñez, como así también que la oferta de atención por parte del sistema público de salud tiene limitaciones que se hacen más notorias en los períodos de alta demanda asistencial, cuando concurren de forma simultánea factores de riesgo como las infecciones virales, el frío y la contaminación aérea. Se torna entonces indispensable que las autoridades del sector público y privado de salud conozcan la información necesaria sobre los requerimientos de la demanda en los distintos grupos de edad, su distribución estacional y su evolución en el tiempo, como elementos críticos para tomar decisiones correctas en la asignación y focalización de los recursos. 
Desde 1992, el Programa Nacional de Infecciones Respiratorias Agudas (IRA) del Ministerio de Salud de Chile creó un sistema de registro prospectivo denominado "monitoreo epidemiológico", cuyo desarrollo se refiere a tres objetivos fundamentales: a) investigar la estructura interna de las consultas pediátricas en atención primaria, en especial las de causa respiratoria, y su evolución temporal; b) construir una base de datos con esta información a fin de tenerla disponible para estudiar eventuales factores de riesgo que pudieran estar relacionados con las variaciones en la frecuencia, cantidad y calidad de estas enfermedades, y c) disponer de un sistema que preste utilidad a las autoridades como vigilancia epidemiológica (8).

El presente trabajo se propone describir la metodología de análisis y la distribución global de las consultas de morbilidad - particularmente las de tipo respiratorio- en menores de 15 años de edad de la ciudad de Santiago, con el objetivo de conocer sus diferencias según grupos etarios, su comportamiento estacional y su evolución a lo largo de los años.

\section{MATERIALES Y MÉTODOS}

Se realizó un estudio de tipo descriptivo con información recogida en forma prospectiva. Considerando que en Santiago funcionan más de 100 centros de APS y teniendo en cuenta el alto número de cuadros respiratorios que se producen diariamente, para que la vigilancia de estas enfermedades fuese oportuna resultó necesario elegir algunos centros cuyos datos se pudieran revisar diariamente y que al mismo tiempo representaran el comportamiento de la totalidad de ellos. Con esta idea fueron seleccionados siete centros de APS representativos de los cuatro puntos cardinales de la provincia de Santiago, mediante la metodología de "centros centinela" propuesta por UNICEF (9). Estos centros fueron: "Avendaño" de la comuna de Lo Prado, “Centro de Salud No 5" de Santiago, "Dr. Anibal Ariztía" de Las Condes, "Joao Goulart" de La Granja, "La Feria" de Pedro Aguirre Cerda, "Lo Barnechea" de la comuna de Lo Barnechea y "Villa O'Higgins" de La Florida. En todos ellos funcionaba una "sala de hospitalización abreviada (sala IRA)", unidad operativa del Programa de IRA de Chile, instaladas en Chile en $1990(8,10)$. Los centros

CUADRO 1. Ubicación de centros centinela (CC), por comuna, servicio de salud y población inscrita 0 asignada, Santiago de Chile, Chile

\begin{tabular}{llcr}
\hline \multicolumn{1}{c}{ Establecimiento } & \multicolumn{1}{c}{ Comuna } & Servicio metropolitano & Población $(n)$ \\
\hline Avendaño & Lo Prado & Occidente & 30019 \\
Centro de Salud No. 5 & Santiago & Central & 45597 \\
Dr. Aníbal Ariztía & Las Condes & Oriente & 43312 \\
João Goulart & La Granja & Suroriente & 31960 \\
La Feria & Pedro Aguirre Cerda & Sur & 28869 \\
Lo Barnechea & Lo Barnechea & Oriente & 36153 \\
Villa O'Higgins & La Florida & Suroriente & 39482 \\
Población total en CC & & & 255392 \\
Población total en provincia de Santiago & & 3365515 \\
Representación de los CC (\%) & & 7,6 \\
\hline
\end{tabular}

Fuente: elaboración de los autores.

centinela incorporados a este sistema de registro, así como su ubicación y pertenencia, se muestran en el cuadro 1. En la actualidad existen 536 salas IRA, distribuidas en los 29 servicios de salud del país. La población asignada a los siete centros no presentó variaciones significativas durante el período del estudio.

Los profesionales que se desempeñan en las salas IRA son un médico con formación en neumología pediátrica y un kinesiólogo, especialmente capacitado en enfermedades respiratorias. Este último profesional es el responsable de la sala de hospitalización abreviada y el encargado de la programación y ejecución de las actividades asistenciales y educacionales sobre IRA, junto al resto del equipo de salud del centro de APS. En cada centro, el personal involucrado en el estudio incluyó al kinesiólogo a cargo de la respectiva sala, además de un médico y una o dos personas del área administrativa. Dicho equipo fue permanentemente informado, capacitado y supervisado en el terreno, con el objetivo de minimizar sesgos al momento de efectuar los registros y enviarlos al equipo de investigadores del nivel central, para su posterior consolidación y análisis. En este nivel, un equipo de tres profesionales, aparte del personal administrativo, se mantuvo en contacto periódico con cada uno de los centros desde donde procedía la información, con objeto de controlar la calidad de los registros y de las comunicaciones, así como del exacto ingreso de la información a un sistema de base de datos.

A fin de disponer de información estandarizada y periódica, se elaboró un formulario para la recolección y transmisión de la información diaria y resumida de los diagnósticos, además de un instructivo para la clasificación de las en- fermedades respiratorias cuyo propósito fue interpretar la forma como efectúan el diagnóstico los médicos y convertirla en datos que pudieran incorporarse al formulario ad-hoc. Se consideró IRA alta a los siguientes diagnósticos: rinofaringitis, resfrío común, faringitis, adenoiditis, rinitis aguda, rinitis purulenta, sinusitis, rinosinusitis, virosis respiratoria, faringoamigdalitis, amigdalitis aguda, amigdalitis pultácea, absceso amigdaliano, otitis media aguda, otitis catarral, faringootitis, rinofaringootitis y otitis supurada. Asimismo, fue considerada como IRA baja a los diagnósticos de síndrome bronquial obstructivo (SBA, SBOR, BOR, $\mathrm{AB}$, bronquitis obstructiva, bronquiolitis, asma bronquial), neumonía (BN, BRN, síndrome neumónico, bronconeumonía, neumopatía) y otras IRA bajas como laringitis aguda, LOA, laringitis catarral, rinofaringolaringitis, traqueitis, laringotraqueitis, faringobronquitis, laringobronquitis, traqueobronquitis, bronquitis aguda, bronquitis catarral, neumonitis, síndrome coqueluchoideo, coqueluche, tos convulsiva, tos ferina, gripe, estado gripal, influenza y atelectasia.

Mediante estos instrumentos, se recolectó prospectivamente la información diaria de todas las consultas de menores de 15 años, especificando el diagnóstico cuando era de tipo respiratoria. Así, las $\mathrm{CP}$ se agruparon en: consulta de causa no respiratoria (CNR), infección respiratoria aguda (IRA), infección respiratoria aguda de vía aérea alta (IRAa), infección respiratoria aguda de vía aérea baja (IRAb), síndrome bronquial obstructivo (SBO) y neumonía. Solo fueron consideradas las consultas de morbilidad, excluyéndose las de control de niño sano, y no se estableció un sistema para diferenciar la primera consulta de cada episodio de enfermedad; es decir, se trata consultas y 
no de casos de enfermedad. Tampoco hay diferenciación de las CNR. La estratificación por grupo etario (menores de 1 año, 1 a 4 años y 5 a 14 años) se incorporó al registro a partir de 1997.

Las comunicaciones para el envío de datos se realizaron los primeros años por vía de fax, lo que permitió obtener registros con un retraso no mayor de 48 horas, y posteriormente por correo electrónico, lo que hizo que la información se recibiera antes de 24 horas. Dado que el número total diario de consultas en APS no es elástico, sino que tiene un límite que está dado por la cantidad de horas médicas disponibles para la atención, los resultados no se expresan en tasas por población sino como proporción del total de consultas, para cada grupo de enfermedades, en datos mensuales o anuales según el caso.

\section{RESULTADOS}

Desde el 4 de enero de 1993 hasta el 31 de diciembre de 2009 se registraron 1947477 consultas en menores de 15 años de edad. De este total, 1188029 $(61,0 \%)$ fueron por causa respiratoria: 656567 por IRAb (33,7\%), 418932 $(21,5 \%)$ por SBO y 48669 por neumonía (2,5\%). En el cuadro 2 se pueden ver los resultados generales para los 17 años estudiados.

Al agrupar los datos en promedios mensuales se puede observar que, con respecto al total de $\mathrm{CP}$, las consultas totales por IRA variaron entre un máximo de $79,62 \%$ en junio de 2009 y un mínimo de $38,94 \%$ en febrero de 2008; el resto fueron CNR. La IRAb alcanzó un

CUADRO 2. Distribución de consultas pediátricas según diagnóstico, Santiago de Chile, Chile, enero de 1993-diciembre de 2009

\begin{tabular}{lrr}
\hline Diagnóstico & \multicolumn{1}{c}{$n$} & \multicolumn{1}{c}{$\%$} \\
\hline CNR & 759448 & 39,0 \\
CR & 1188029 & 61,0 \\
$\quad$ Total & 1947477 & 100,0 \\
& & \\
IRAa & 531462 & 27,3 \\
IRAb & 656567 & 33,7 \\
$\quad$ Total & 1188029 & 61,0 \\
SBO & 418932 & \\
Neumonía & 48669 & 21,5 \\
Otras IRAb & 188966 & 2,5 \\
& & 9,7 \\
\hline
\end{tabular}

Fuente: elaboración de los autores.

CNR: consultas no respiratorias; CR: consultas respiratorias; IRAa: infección respiratoria aguda de vía aérea alta; IRAb: infección respiratoria aguda de vía aérea baja; SBO: síndrome bronquial obstructivo. máximo de 53,95\% en julio de 2001 y un mínimo de $15,19 \%$ en febrero de 2008. El diagnóstico de SBO osciló entre 33,01\% en julio de 1993 y 9,62\% en febrero de 2009. La neumonía varió entre $7,15 \%$ en junio de 1996 y 0,48\% en febrero de 2004.

Con respecto a la tendencia observada a lo largo de todo el período, al aplicar a los datos anuales un análisis de regresión en series de tiempo, se ve una mantención en la proporción de IRA totales, pero una reducción significativa en el porcentaje de consultas por IRAb $(0,64 \%$ anual; $P<0,001)$, neumonía $(0,099 \% ; P<0,001)$ y síndrome bronquial obstructivo $(0,33 \% ; P<0,001)$ (cuadro 3). Para 1997-2009 se puede calcular la distribución de las enfermedades por grupos etarios. En este período el total de CP fue de 1409516 , de las cuales
278895 fueron en menores de 1 año, 539988 en el grupo de 1 a 4 años y 590633 en el de 5 a 14 años (cuadro 4).

Los resultados anteriores pueden ser expresados en función de mínimos, máximos y medianas para las consultas de IRAb durante los últimos siete años, lo cual permite construir "canales endémicos" que puedan orientar al lector respecto de las características de un año normal de consultas. Los canales endémicos para 2003-2009 se presentan en las figuras 1, 2, 3 y 4 .

\section{DISCUSIÓN}

La ventaja de usar la modalidad de "centros centinela" reside en que facilita una recolección de información cualitativa y cuantitativa sobre un determinado

CUADRO 3. Proporción de consultas por diagnóstico y por año en centros centinela de atención primaria, Santiago de Chile, Chile, 1993-2009

\begin{tabular}{lcccccc}
\hline \multicolumn{7}{c}{ Diagnóstico } \\
Año & CNR & CR & IRAa & IRAb & SBO & Neumonía \\
\hline \cline { 2 - 6 } 1993 & 37,44 & 62,56 & 25,28 & 37,28 & $22,82(61,21)$ & $3,40(9,12)$ \\
1994 & 37,38 & 62,62 & 24,96 & 37,66 & $23,03(61,15)$ & $3,22(8,55)$ \\
1995 & 39,14 & 60,86 & 23,79 & 37,08 & $22,28(60,09)$ & $3,48(9,39)$ \\
1996 & 39,23 & 60,77 & 23,30 & 37,47 & $22,61(60,34)$ & $3,40(9,07)$ \\
1997 & 39,82 & 60,18 & 25,00 & 35,17 & $22,84(64,92)$ & $2,79(7,94)$ \\
1998 & 39,53 & 60,47 & 26,37 & 34,09 & $22,83(66,97)$ & $2,38(6,97)$ \\
1999 & 40,96 & 59,04 & 25,00 & 34,05 & $22,95(67,41)$ & $2,10(6,16)$ \\
2000 & 37,76 & 62,24 & 29,41 & 32,83 & $21,15(64,42)$ & $2,11(6,44)$ \\
2001 & 36,55 & 63,45 & 27,81 & 35,64 & $22,84(64,09)$ & $2,49(7,00)$ \\
2002 & 37,64 & 62,36 & 28,04 & 34,32 & $20,54(59,85)$ & $2,29(6,68)$ \\
2003 & 40,49 & 59,51 & 27,23 & 32,28 & $21,04(65,17)$ & $1,68(5,19)$ \\
2004 & 39,96 & 60,04 & 28,57 & 31,48 & $21,05(66,88)$ & $1,85(5,87)$ \\
2005 & 37,35 & 62,65 & 28,70 & 33,95 & $23,04(67,85)$ & $2,60(7,65)$ \\
2006 & 40,09 & 59,91 & 31,19 & 28,72 & $19,91(69,33)$ & $2,12(7,37)$ \\
2007 & 40,69 & 59,31 & 30,05 & 29,26 & $19,29(65,93)$ & $2,17(7,41)$ \\
2008 & 42,75 & 57,25 & 30,81 & 26,44 & $17,33(65,55)$ & $1,59(6,03)$ \\
2009 & 39,63 & 60,37 & 33,11 & 27,26 & $15,59(57,19)$ & $1,90(6,96)$ \\
Media & 38,96 & 61,04 & 27,22 & 33,82 & $21,63(63,96)$ & $2,51(7,42)$ \\
\hline
\end{tabular}

Fuente: elaboración de los autores.

CNR: consultas no respiratorias; CR: consultas respiratorias; IRAa: infección respiratoria aguda de vía aérea alta; IRAb: infección respiratoria aguda de vía aérea baja; SBO: síndrome bronquial obstructivo

Nota: entre paréntesis se presenta el porcentaje de consultas por determinado diagnóstico, con respecto al total de consultas por IRAb, para cada año. .

CUADRO 4. Proporción de consultas pediátricas por diagnóstico y grupo etario en Santiago de Chile, Chile, 1997-2009

\begin{tabular}{|c|c|c|c|c|c|c|c|c|}
\hline \multirow[b]{3}{*}{ Diagnóstico } & \multicolumn{8}{|c|}{ Grupos de edad (años) } \\
\hline & \multicolumn{2}{|c|}{$<1$} & \multicolumn{2}{|c|}{$1-4$} & \multicolumn{2}{|c|}{$5-14$} & \multicolumn{2}{|c|}{$0-14$} \\
\hline & No. & $\%$ & No. & $\%$ & No. & $\%$ & No. & $\%$ \\
\hline IRAa & 70657 & 25,33 & 160434 & 29,71 & 168850 & 28,59 & 399941 & 28,37 \\
\hline IRAb & 113743 & 40,78 & 203058 & 37,6 & 138656 & 23,48 & 455457 & 32,31 \\
\hline SBO & 86083 & 30,87 & 135178 & 25,03 & 75456 & 12,78 & 296717 & 21,05 \\
\hline Neumonía & 8527 & 3,06 & 14840 & 2,75 & 7193 & 1,22 & 30560 & 2,17 \\
\hline Total & 278895 & 100,00 & 539988 & 100,00 & 590633 & 100,00 & 1409516 & 100,00 \\
\hline
\end{tabular}

Fuente: elaboración de los autores.

IRAa: infección respiratoria aguda de vía aérea alta; IRAb: infección respiratoria aguda de vía aérea baja; SBO: síndrome bronquial obstructivo. 
FIGURA 1. Proporción de consultas por infección respiratoria aguda baja en menores de 15 años de edad, con respecto al total de consultas pediátricas, Santiago de Chile, Chile, 2003-2009

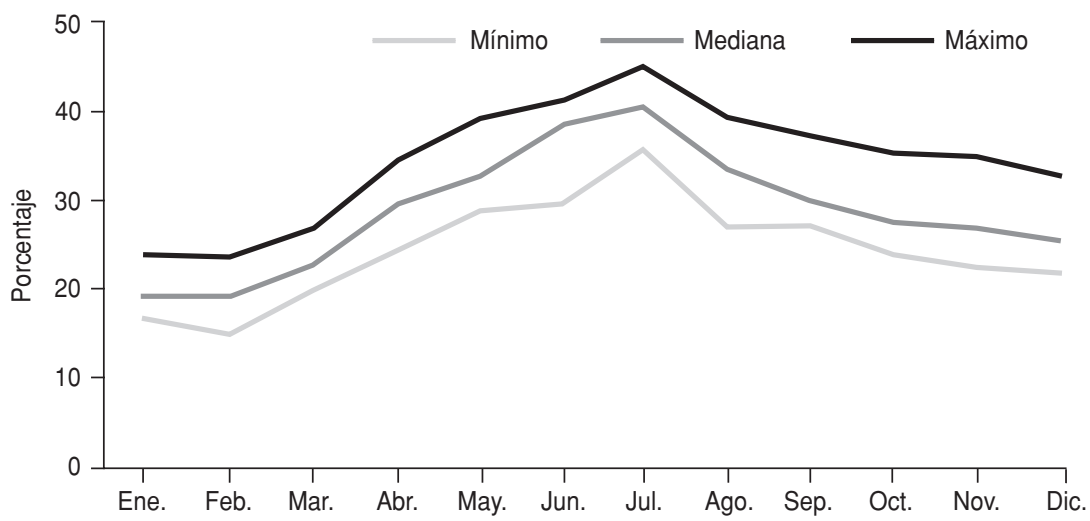

FIGURA 2. Proporción de consultas por infección respiratoria aguda baja en menores de 1 año de edad, con respecto al total de consultas pediátricas, Santiago de Chile, Chile, 2003-2009

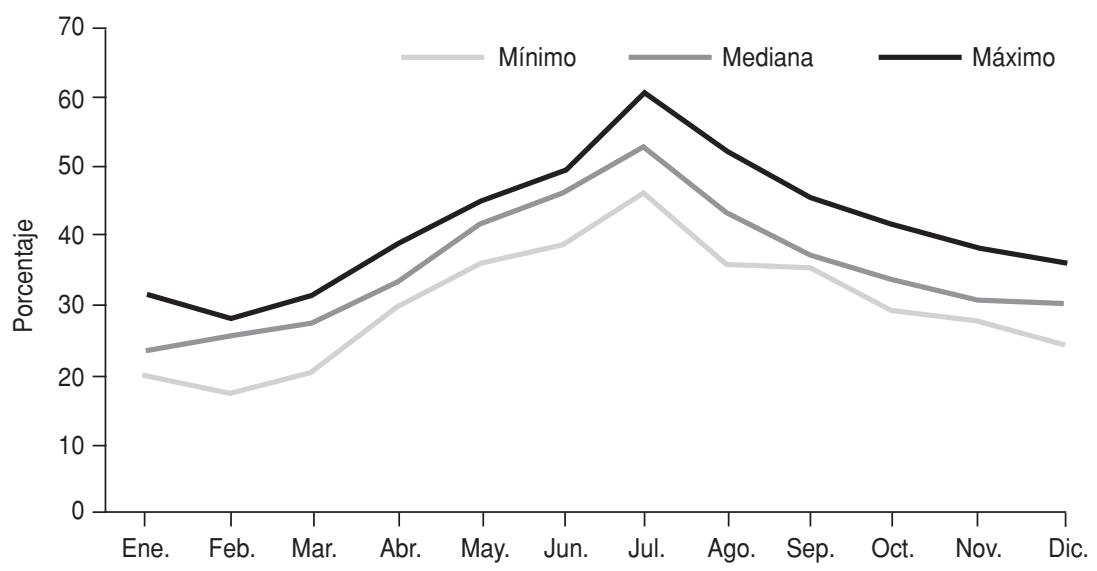

FIGURA 3. Proporción de consultas por infección respiratoria aguda baja en niños de 1 a 4 años de edad, con respecto al total de consultas pediátricas de ese grupo, Santiago de Chile, Chile, 2003-2009

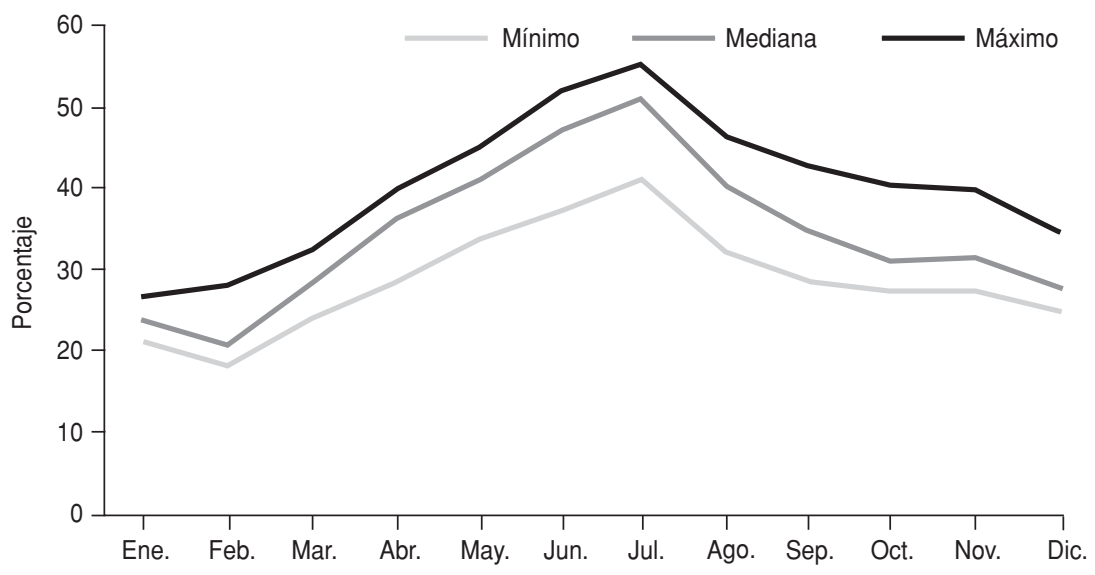

número de variables en forma rápida, flexible, sostenida en el tiempo, a bajo costo y con la participación de los actores involucrados en el nivel local (9).
Además permite seleccionar grupos representativos del universo en estudio, en muestra por conglomerados, que pueden ser de gran utilidad para manejar distin- tos problemas prioritarios de salud, convirtiéndola en una herramienta insustituible para gestionar adecuadamente las medidas de salud pública que requieren ser implementadas según la estacionalidad que presentan estas enfermedades.

Los centros de salud seleccionados permitieron observar un $7,6 \%$ del total de la población de la provincia de Santiago (11). Al respecto, cabe destacar la representatividad de la muestra, pues la mayoría de los estudios previos se realizaron en uno o dos centros o servicios de salud o por cortos períodos de tiempo. La representación geográfica consideró los distintos sectores de la provincia de Santiago, dando cuenta de las probables peculiaridades existentes al interior de su cuenca, sometida a los efectos $-\mathrm{y}$ las características topoclimatológicas- de la capa de inversión térmica (12).

Con respecto a los diagnósticos, si bien están basados en parámetros preferentemente clínicos, la calidad de la información está avalada por el hecho de que todos fueron realizados por médicos, y contaron con la presencia de un kinesiólogo capacitado y familiarizado con la nomenclatura usada en enfermedades respiratorias, el uso de formularios estandarizados, el control de la calidad de los registros, la asesoría técnica permanente de los investigadores al equipo local y las visitas al terreno.

En cuanto a los resultados generales, se reafirma la impresión de que las enfermedades respiratorias constituyen la primera causa de consultas pediátricas en atención primaria y que incluso en verano dan cuenta de casi la mitad de ellas. La proporción de consultas respiratorias con respecto al total de consultas pediátricas alcanza valores similares $\mathrm{y}$, en algunos casos, aun superiores a los mostrados en investigaciones nacionales previas para los mismos grupos etarios. Lo anterior es importante si se repara en que este sistema de vigilancia en la Región Metropolitana hizo posible estudiar casi 2 millones de CP, cifra largamente superior a las consideradas en estudios anteriores (1, 5-7).

Asimismo, a lo largo del tiempo se observa una disminución significativa en el porcentaje de consultas por IRAb, neumonía y SBO. Las probables explicaciones de este fenómeno son las estrategias de prevención, promoción y manejo clínico oportuno y precoz implementadas por el Programa IRA a partir de 1990. Las actividades de este programa incluyeron per- 
FIGURA 4. Proporción de consultas por infección respiratoria aguda baja en niños de 5 a 14 años de edad, con respecto al total de consultas pediátricas de ese grupo, Santiago de Chile, Chile, 2003-2009

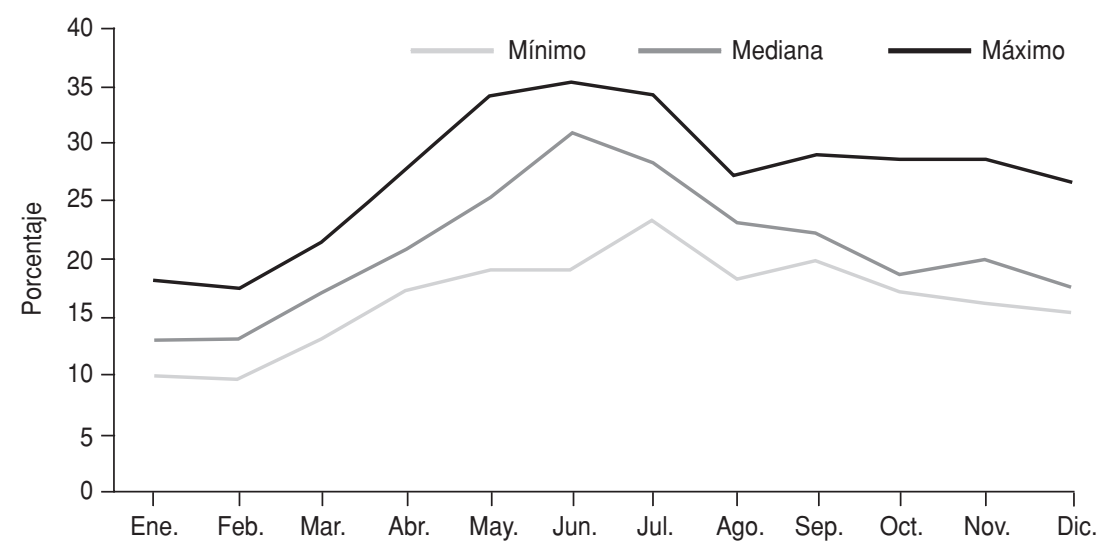

manentemente educación a la población en el conocimiento y prevención de factores de riesgo de enfermar por causa respiratoria - y en la gravedad de las IRAa fin de consultar en forma oportuna. Además se utilizaron normas técnicas protocolizadas y disponibilidad completa de fármacos precisamente para permitir el cumplimiento de dichas normas.

Con respecto a los resultados por edad, se puede ver que la proporción de consultas por cuadros de mayor gravedad, como neumonía y SBO, es claramente mayor en los menores de 5 años $\mathrm{y}$, sobre todo, en los menores de 1 año. Esto confirma que los menores de un año, proporcionalmente, consultan con mayor frecuencia y por diagnósticos de mayor severidad que los grupos de mayor edad. Es también destacable que las consultas por IRAb en el grupo de menores de 5 años dan cuenta de casi $70 \%$ del total de consultas por dicha causa para el período 1997-2009.

Se halló también un alto porcentaje de visitas médicas por SBO, cuadro que aparece nítidamente como la primera causa específica de consulta, coincidiendo con lo reflejado en estudios anteriores (2-4, 6, 13). Esto obliga a reforzar la capacitación y la asignación de recursos dirigidos a este síndrome de alta relevancia epidemiológica. Como ya se ha reportado, la marcada estacionalidad de las IRA se relaciona directamente con factores de riesgo, incluidos los altos niveles de contaminación atmosférica presentes durante los meses de invierno (13).

Dado que el presente estudio fue realizado en centros de salud de atención primaria pertenecientes al sistema público, que cubre a $77 \%$ de la población, el quintil de nivel socioeconómico más alto de la población no necesariamente queda representado en este análisis. Otra de las posibles limitaciones radica en que, tal como ha sido descrito, en la metodología de registros utilizada se consigna el número de consultas y no el número de episodios de enfermedad, y por lo tanto no se puede calcular incidencia. Tal limitación debería ser más relevante en el análisis de casos de neumonía, dado que un paciente podría haber consultado más de una vez por episodio por tratarse de un cuadro más grave.
Por otro lado, la expresión de los resultados en porcentajes y no en otro tipo de tasas se explica porque la oferta total de consultas carece de elasticidad, es decir que al aumentar la demanda las consultas pueden no aumentar aunque sí variar su composición, por dirigirse la oferta hacia la atención de los cuadros más severos, en desmedro de los más leves. Este hecho, y la necesidad de utilizar la información como sistema de vigilancia, hacen necesario expresar los datos en proporciones, recogiendo de esta manera en forma más representativa y oportuna las variaciones de la demanda de morbilidad.

En síntesis, el presente trabajo ofrece al lector una descripción de los beneficios y desventajas del "sistema de centros centinela", mediante el cual se pudieron caracterizar las consultas pediátricas, su comportamiento estacional y su distribución en menores de 15 años de edad. Esta modalidad de recolección y análisis de la información puede ser útil para disponer de un sistema de vigilancia de enfermedades respiratorias en países con similar organización sanitaria que la chilena, pues es una valiosa herramienta para la gestión de las intervenciones sanitarias que se desarrollan en este campo. Se confirma que las IRA constituyen la primera causa de consultas pediátricas en atención primaria, que incluso en los meses de verano dan cuenta de casi la mitad de las visitas médicas de este grupo etario y que los menores de un año consultan con mayor frecuencia $-\mathrm{y}$ por diagnósticos de mayor severidad. En virtud de estos resultados, es perentorio que las autoridades sanitarias del sector público y privado dispongan de esta información para poder asignar o reasignar recursos en programas de promoción, educación, prevención y tratamiento de las enfermedades respiratorias en niños menores de 15 años de edad.

\section{REFERENCIAS}

1. Segovia I, Staab K, Orrego A. Estudio de prevalencia de las enfermedades respiratorias infantiles en el consultorio Dr. Luis Sepúlveda en 1989. Cuad Med Soc. 1992;33(2):81-6.

2. Ubilla C, Olivari F, Ceruti E, San Martín E, Sciaraffia F, Silva P. Síndrome bronquial obstructivo $(\mathrm{SBO})$ en la consulta pediátrica ambulatoria. Pediatria (Santiago de Chile). 1989; 32(2):81-4.
3. López I, Sepúlveda H, Gelman M. Síndrome bronquial obstructivo: otro problema del nivel primario. Pediatria (Santiago de Chile). 1991;34(2):76-80.

4. López I, Sepúlveda H, Valdés I. Síndrome bronquial obstructivo en los primeros 4 años de vida. Rev Chil Salud Publica. 1997;1(1):9-15.

5. López I, Sepúlveda H, Nazar R, Guerra J, Valle P. Perfil de morbilidad anual de meno- res de 6 años consultantes. Rev Chil Pediatr. 2000;71(4):321-7.

6. López I, Sepúlveda H, Nazar R, Martínez W, Pacheco P, Montero A. Infección respiratoria aguda baja del niño en atención primaria. Rev Chil Pediatr. 2001; 72(3):204-11.

7. Aranda C, Astudillo P, Mancilla P, Caussade S, Girardi G. Caracterización epidemiológica de las consultas pediátricas por causa respira- 
toria en atención primaria en Chile. OPS, Serie HCT/AIEPI-3E. 1998; I: 43-9.

8. Girardi G, Astudillo P, Zúñiga F. El Programa IRA en Chile: Hitos e historia. Rev Chil Pediatr. 2001;72(4):292-300.

9. UNICEF. Sitios centinelas: monitoreo y evaluación. Nueva York: UNICEF; 1991. Pp. 1-8.

10. Astudillo P, Mancilla P, Girardi G, Aranda C, Gamboa R. Hospitalización abreviada en atención primaria de salud. Rev Chil Enf Respir. 1992;8(supl 1): 262.
11. Instituto Nacional de Estadísticas (INE), Chile. Proyecciones de población. Disponible en: http://www.ine.cl/canales/chile_estadis tico/demografia_y_vitales/demo_y_vita. php Acceso el 12 de mayo de 2012.

12. Aranda C, Romero H. Topoclimatología de la cuenca de Santiago y sus efectos en la contaminación atmosférica y en la salud. Enf Resp Cir Tor. 1989:5:24-30

13. Prieto MJ, Mancilla P, Astudillo P, Reyes A, Román O. Exceso de morbilidad respi- ratoria en niños y adultos mayores en una comuna de Santiago con alta contaminación atmosférica por partículas. Rev Med Chile. 2007;135(2):221-8.

Manuscrito recibido el 11 de octubre de 2011. Aceptado para publicación, tras revisión, el 18 de abril de 2012.
ABSTRACT

\section{Epidemiology of pediatric respiratory consultations in Santiago de Chile, from 1993 to 2009}

Key words
Objective. This paper describes the analytical methodology and overall distribution of these consultations-particularly respiratory consultations-for children under 15 years of age in Santiago de Chile, Chile. The aim is to understand differences by age groups, as well as seasonal trends and trends over the years.

Methods. The research covered the distribution of consultations and their evolution over a period of 17 years. Information was collected on a daily basis from all pediatric consultations, grouped into nonrespiratory and acute respiratory categories, in seven sentinel centers of Santiago de Chile.

Results. Between January 1993 and December 2009, 1947477 cases of pediatric illnesses were recorded, of which 1188029 (61.0\%) were for respiratory causes. Of those, $656567(33.7 \%)$ were for acute lower respiratory tract illnesses, $418932(21.5 \%)$ were for broncho-obstructive syndrome, and 48669 (2.5\%) were for pneumonia. Pneumonia and broncho-obstructive syndrome were more frequent in children under age 5. Lower respiratory tract illnesses, broncho-obstructive syndrome, and pneumonia showed a significant downward trend during the period observed. Lower respiratory tract illnesses are the leading cause of pediatric morbidity in primary health care, while broncho-obstructive syndrome is the leading specific reason for pediatric consultations.

Conclusions. These results point to the need to allocate or reallocate resources for programs for promotion, education, prevention, and treatment of these illnesses with the targeting necessary to address seasonal variations.

Respiratory tract infections; epidemiological surveillance; pediatrics; child health (public health); Chile. 